\title{
Konsensusempfehlung zur strukturierten Befunderstellung onkologischer PET-Hybridbildgebung*
}

\section{Structured reporting in oncologic hybrid imaging: a consensus recommendation}

\author{
Autoren \\ Thorsten Derlin ${ }^{1}$, Sergios Gatidis ${ }^{2}$, Bernd Joachim Krause ${ }^{3}$, Gerald Antoch ${ }^{4}$, Jörg Kotzerke ${ }^{5}$, Daniel Pinto dos Santos ${ }^{6}$, \\ Matthias Eiber ${ }^{7}$, Marc-André Weber ${ }^{8}$, Frederik Giesel $^{9}$, Christina Pfannenberg ${ }^{2}$, Heinz-Peter Schlemmer ${ }^{10}$, \\ Thorsten Persigehl ${ }^{6}$, Ken Herrmann ${ }^{11}$, Lale Umutlu ${ }^{12}$ \\ Vorstand der Interdisziplinären AG für Hybride Bildgebung in alphabetischer Reihenfolge: \\ Gerald Antoch ${ }^{4}$, Ken Herrmann ${ }^{11}$, Bernd Joachim Krause ${ }^{3}$, Lale Umutlu ${ }^{12}$ \\ Vorstand der Deutschen Röntgengesellschaft (DRG) und der Deutschen Gesellschaft für Nuklearmedizin (DGN) in \\ alphabetischer Reihenfolge: \\ Gerald Antoch ${ }^{4}$, Frank Anton ${ }^{13}$, Jörg Barkhausen ${ }^{14}$, Peter Bartenstein ${ }^{15}$, Bernd Joachim Krause ${ }^{3}$, Markus Luster $^{16}$, \\ Stefan Neumann ${ }^{13}$, Stefan 0. Schönberg ${ }^{17}$
}

Institute

1 Department of Nuclear Medicine, Hannover Medical School, Hannover, Germany

2 Department of Diagnostic and Interventional Radiology, University Hospital Tuebingen, Tuebingen, Germany

3 Department of Nuclear Medicine, University Medical Center Rostock, Rostock, Germany

4 Department of Diagnostic and Interventional Radiology, University Duesseldorf, Medical Faculty, Duesseldorf, Germany

5 Department of Nuclear Medicine, Faculty of Medicine and University Hospital Carl Gustav Carus, TU Dresden, Germany

6 Institute of Diagnostic and Interventional Radiology, University Hospital of Cologne, Cologne, Germany

7 Department of Nuclear Medicine, Technical University of Munich, Munich, Germany

8 Institute of Diagnostic and Interventional Radiology, Pediatric Radiology and Neuroradiology, University Medical Center Rostock, Rostock, Germany

9 Department of Nuclear Medicine, Heidelberg University Hospital, Heidelberg, Germany

10 Department of Radiology, German Cancer Research Center (DKFZ), Heidelberg, Germany

11 Department of Nuclear Medicine, University Hospital Essen, Essen, Germany

12 Department of Diagnostic and Interventional Radiology and Neuroradiology, University Hospital Essen, Essen, Germany

13 Deutsche Röntgengesellschaft, Berlin, Germany
14 Department of Radiology and Nuclear Medicine, University Hospital Schleswig-Holstein, Campus Lübeck, Lübeck, Germany

15 Department of Nuclear Medicine, University Hospital, LMU Munich, Munich, Germany

16 Department of Nuclear Medicine, University Hospital Marburg, Marburg, Germany

17 Department of Clinical Radiology and Nuclear Medicine, University Medical Center Mannheim, Germany

Für den Vorstand der DGN

Prof. Dr. Bernd Joachim Krause, Präsident

Prof. Dr. Markus Luster, stellvertretender Präsident

Prof. Dr. Peter Bartenstein, Schriftführer

Für den Vorstand der DRG

Prof. Dr. Gerald Antoch, Präsident

Prof. Dr. Stefan O. Schönberg, stellvertretender Präsident

Prof. Dr. Jörg Barkhausen, President-elect

Dr. Frank Anton, Schatzmeister

Dr. Stefan Neumann, Schriftführer

Schlüsselwörter

Strukturierte Befundung, Onkologie, Befundungsleitfaden, Hybridbildgebung, PET, PET/CT, PET/MRI

Key words

structured reporting, reporting guidance, oncology, hybrid imaging, PET, PET/CT, PET/MRI

eingereicht 08.05 .2020

akzeptiert 11.05.2020 
Bibliografie

DOI https://doi.org/10.1055/a-1176-0275

Nuklearmedizin 2020; 59: 288-293

(c) Georg Thieme Verlag KG, Stuttgart · New York

ISSN 0029-5566

Korrespondenzadresse

Prof. Dr. med. Thorsten Derlin

Sektion Translationale onkologische Nuklearmedizin

Klinik für Nuklearmedizin, Medizinische Hochschule Hannover

(MHH), Carl-Neuberg-Str. 1, 30625 Hannover, Deutschland

Tel.: ++ 49/511/5322579

Fax: $++49 / 511 / 5323761$

Derlin.Thorsten@mh-hannover.de

Supplemental Material:

https://doi.org/10.1055/a-1176-0275

\section{ZUSAMMENFASSUNG}

Seit Einführung der PET/CT im Jahr 2001 bzw. der PET/MRT im Jahr 2010 ist die PET als Hybridbildgebung und Instrument einer bildgesteuerten Präzisionsmedizin zunehmend zu einem Versorgungsstandard in der Onkologie geworden. Die zentrale Schnittstelle in der Kommunikation zwischen den zuweisenden onkologisch tätigen Ärzten und dem Nuklearmediziner und Radiologen stellt der schriftliche Befund dar, der das Patientenmanagement und Outcome beeinflusst, aber auch juristisch-relevante Dokumentation der erbrachten Leistung und ärztlichen Beurteilung der erhobenen Befunde ist. Eine qualitativ hochwertige, auf die klinische Frage fokussierte Hybridbildgebung sollte im weiteren Fortgang mit einem gleichermaßen qualitativ hochwertigen, strukturierten schriftlichen Befund verbunden sein, der die klinische Frage zur Zufriedenheit des zuweisenden Arztes beantwortet. Im vorliegenden Manuskript werden daher Konsensempfehlungen zur Befundstruktur, zu Befundinhalten und zur konklusiven Beurteilung einer onkologischen Hybridbildgebung gegeben. Darüber hinaus werden beispielhafte Vorlagen für ebensolche Befunde dargestellt. Die hier dargelegten Empfehlungen zur strukturierten Befunderstellung sollen eine weitere Standardisierung und Harmonisierung onkologischer Befunde im Kontext einer Hybridbildgebung fördern und so die Kommunikation mit den zuweisenden Ärzten vereinfachen und die Akzeptanz und Wertschätzung der onkologischen Hybridbildgebung weiter unterstützen.

\section{ABSTRACT}

Since the clinical introduction of PET/CT in the year of 2001 and PET/MRI in the year of 2010, hybrid imaging-guided precision medicine has become an important component of diagnostic algorithms in oncology. The written report represents the primary mode of communication between the referring physician and both the nuclear medicine physician and the radiologist. Reports have considerable impact on patient management and patient outcome, and serve as a legal documentation of the services provided and the expert impression of the interpreting physician. A high-quality hybrid imaging study should result in a likewise high-quality, structured written report which satisfactorily answers the clinical question of the referring physician. In this manuscript, consensus recommendations for structure and content of oncologic hybrid imaging reports and conclusive impressions are provided. Moreover, exemplary structured reports are provided. The recommendations for structured reporting provided in this document should foster further standardization and harmonization of oncologic reports in the context of hybrid imaging. They should also simplify communication with referring physicians and support both acceptance and appreciation of the clinical value of oncologic hybrid imaging.

\section{Citation Format}

- Derlin T, Gatidis S, Krause BJ et al. Konsensusempfehlung zur strukturierten Befunderstellung onkologischer PETHybridbildgebung. Nuklearmedizin 2020; 59 :

DOI:10.1055/a-1176-0275

\section{Einleitung}

Methoden der klinischen Hybridbildgebung, worunter in diesem Manuskript die Positronen-Emissions-Tomographie/Computertomographie (PET/CT) und die Positronen-Emissions-Tomographie/ Magnetresonanztomographie (PET/MRT) zusammengefasst werden, sind fester Bestandteil der klinisch-onkologischen diagnostischen Algorithmen, stellen den Bildgebungsstandard für eine Reihe onkologischer Erkrankungen dar und ermöglichen eine bildgeführte Präzisionsmedizin [1]. Die Integration zweier Bildgebungsmodalitäten mit unterschiedlichem Informationsgehalt führt dabei zu inhaltlichen und organisatorischen Herausforderungen, die in vielen Aspekten spezifisch für die Hybridbildgebung sind und adressiert werden müssen, um eine optimale Befundqualität im Sinne des Patienten zu erreichen.
Zu Fragen der technischen Durchführung existieren sowohl für die PET/CT als auch die PET/MRT Leitlinien bzw. interdisziplinäre Konsensusempfehlungen nationaler und internationaler Fachgesellschaften, wodurch eine klinische Standardisierung und Minimalanforderungen an die Untersuchungsqualität erreicht werden [2-6].

Bei der Auswertung, Befundung und Interpretation der klinischen Hybridbildgebung kann hingegen eine Heterogenität zwischen verschiedenen Standorten und Fachabteilungen festgestellt werden [7-10]. Bereits wenige Jahre nach klinischer Einführung der PET/CT stellten die europäischen Fachgesellschaften für Nuklearmedizin und Radiologie fest, dass es keine inhaltliche Trennung zwischen morphologischer Information aus CT oder MRT und molekularer Information der PET geben dürfe; vielmehr sei eine Voreingenommenheit bei der Interpretation von Hybriduntersuchungen aufgrund von Ausbildung und Vorwissen zu vermeiden [2, 3]. Sowohl die europäische als auch die amerikanische 
Fachgesellschaft für Nuklearmedizin geben in ihren Leitlinien bzw. Befundrichtlinien Hinweise zur PET/CT Befundung im onkologischen Kontext $[4,11]$. Trotz existierender Handlungsempfehlungen konnten in verschiedenen Studien Defizite in der praktischen PET/CT-Befundung festgestellt werden, sowohl in Bezug auf Form und Inhalt $[12,13]$, als auch in Bezug auf Organisation und Ausbildung [9, 10].

Einigkeit besteht darin, dass die Komplexität der Hybridbildgebung aufgrund des notwendigen technischen Vorwissens, der komplexen Datenstruktur, der großen Zahl entstehender Bilddaten und der häufig vielschichtigen klinischen Fragestellung im Allgemeinen diejenige anderer nuklearmedizinischer und radiologischer Modalitäten übersteigt. Zudem haben hybride Bildgebungsmethoden einen besonderen diagnostischen Stellenwert, da sie häufig bereits den größtmöglichen bildgebenden Informationsgehalt liefern, wodurch das Untersuchungsergebnis in besonderem Maße konkreten Einfluss auf klinische Entscheidungen hat [14-16] und auch besonders häufig in interdisziplinären Tumorboards präsentiert wird. Strukturierte Befunde erleichtern hier zudem den präsentierenden Nuklearmedizinern und Radiologen die Vorbereitung.

Aus den genannten Punkten ergibt sich die Notwendigkeit einer dedizierten Strategie zur Auswertung und Befundung von Hybriduntersuchungen, die zum einen der Komplexität der Modalitäten gerecht wird und zum anderen die im Einzelfall gestellte klinische Frage adressiert.

Zu diesem Zweck haben - im Rahmen zweier interdisziplinärer Workshops der Arbeitsgemeinschaft Bildgebung in der Onkologie (ABO) der Deutschen Krebsgesellschaft (DKG) - Mitglieder der Interdisziplinären Arbeitsgemeinschaft für Hybride Bildgebung (ID AG Hybride BG) als gemeinsames Gremium der Deutschen Gesellschaft für Nuklearmedizin (DGN) und der Deutschen Röntgengesellschaft (DRG) sowie weitere Vertreter der beiden Fachgesellschaften gemeinsame Empfehlungen zum strukturierten Reporting einer onkologischen Hybridbildgebung erarbeitet.

Ziel dieses Beitrags ist die Beschreibung eines solchen strukturierten Vorgehens bei der interdisziplinären klinischen Befundung von PET/CT und PET/MRT-Untersuchungen. Diese Empfehlungen zur Befundstruktur, zur Befundung und zur Beurteilung sollen als Hilfestellung für Befunder in der Hybridbildgebung dienen, helfen, die Befunderstellung zu standardisieren und Mindestanforderungen an einen Hybridbildgebungsbefund definieren [9]. Eine qualitativ hochwertige Befundung setzt ein Expertenwissen in den jeweiligen angewandten Modalitäten voraus, die durch eine gemeinsame Befundung durch nuklearmedizinische und radiologische Fachärzte bzw. fachkundige dual-ausgebildete Fachärzte erreicht wird [17].

Diese - bewusst nicht abschließenden - Empfehlungen zum strukturierten Reporting einer onkologischen Hybridbildgebung sollten Ausgangspunkt für eine prospektive Validierung der vorgeschlagenen Struktur sein und auf Grundlage zukünftig erhobener Daten geprüft und dann gegebenenfalls weiter modifiziert werden. Hierzu wird die Interdisziplinäre Arbeitsgemeinschaft für Hybride Bildgebung in 5 Jahren eine Überprüfung und Überarbeitung vornehmen. Eine stufenweise weitere Fortentwicklung könnte zudem in Form vorformulierter Textbausteine bzw. kate- gorisierter Diagnosen und Textbausteinen mit Wörtern aus kontrollierten Vokabularien erfolgen.

\section{Inhalt des Befundes}

\section{Identifikationsinformationen}

Der Befund sollte Angaben zu den Patientendaten (z. B. Name, Geburtsdatum, Geschlecht) sowie Art und Datum der durchgeführten Untersuchung enthalten.

\section{Klinische Angaben und Anamnese}

Hier sollen die wesentlichen, den Kontext der Untersuchung definierenden klinischen und anamnestischen Angaben einfließen. Hierzu gehören üblicherweise die zur Untersuchung führende Haupt- oder Verdachtsdiagnose, relevante Nebendiagnosen und eine kurze Darstellung relevanter Vortherapien. Bei Vortherapien, welche das Untersuchungsergebnis beeinflussen können (z. B. Chemotherapie, Strahlentherapie, Hormontherapie) sollte auch der zeitliche Abstand zur Hybriduntersuchung benannt werden. Die Ergebnisse aktueller laborchemischer Tests (z. B. Prostataspezifisches Antigen (PSA)-Wert beim Prostatakarzinom) und/ oder pathologische Befunde können kurz referiert werden. Handelt es sich um eine vom Gemeinsamen Bundesausschuss (G-BA) anerkannte Indikation, sollten die die Untersuchung (und Erstattungsfähigkeit) begründenden Voraussetzungen sich idealerweise aus den klinischen Angaben direkt ableiten (z. B. „Entscheidung über die Durchführung einer laryngoskopischen Biopsie beim Larynxkarzinom, wenn nach Abschluss einer kurativ intendierten Therapie der begründete Verdacht auf eine persistierende Erkrankung oder ein Rezidiv besteht“ [18]). Diese Informationen können chronologisch zur Verfügung gestellt werden. Alternativ kann eine strukturierte Abfrage der klinischen Abgaben erfolgen (z. B. „1. Anamnese/Hauptdiagnose, 2. Aktuelle Therapie und aktuelle Gesundheitssituation, 3. Relevante Nebendiagnosen“).

\section{Fragestellung}

Die klinische Fragestellung stellt den zentralen Kommunikationspunkt zwischen zuweisendem Arzt und dem befundenden Nuklearmediziner und/oder Radiologen dar. Eine klar formulierte, spezifische Frage ist Voraussetzung für eine qualitativ hochwertige, auf diese Frage fokussierte Hybridbildgebung und ebenso Voraussetzung für einen qualitativ hochwertigen, die klinische Frage zur Zufriedenheit des zuweisenden Arztes beantwortenden Befund bzw. Beurteilung.

Anhand dieser Frage ergibt sich die Überprüfung der rechtfertigenden Indikation nach §83 Strahlenschutzgesetz (StrlSchG) und nach $\S 119$ Strahlenschutzverordnung (StrlSchV) für die PET und die CT, wobei im Befund vermerkt werden sollte, dass diese überprüft und gestellt wurde.

\section{Untersuchungstechnik}

Wesentliche Parameter der Untersuchungstechnik sind modalitätsabhängig zu benennen $[4,11]$ : 
PET:

- Angabe zu Art und Aktivität des applizierten Radiopharmazeutikums in $\mathrm{MBq}$

- Applikationsweg und optional -ort

- Angaben zum Aufnahmebeginn nach Applikation

- Untersuchte Körperregion(en)

- Ggf. Angaben zur Patientenlagerung (Rücken- versus Bauchlage; Arme eleviert oder seitlich gelagert), insbesondere falls keine Standardbedingungen

- ggf. Angaben zu Spätaufnahmen

- der Typ des verwendeten Hybridsystems sollte spezifiziert werden, weitere techn. Angaben sind optional

- ggf. Angabe der Zahl und Aufnahmezeit pro Bettposition (min/Bettposition) bzw. des körperregionsabhängigen Tischvorschubs ( $\mathrm{mm} / \mathrm{s}$ )

- ggf. Angaben zum Rekonstruktionsalgorithmus (z. B. Iterationen)

- Angaben zur quantitativen Auswertung der schwächungskorrigierten Bilder mittels Standardized Uptake Value (SUV)Berechnung (Angabe der Normierungsmethode)

- ggf. Nüchternblutzucker (bei Untersuchungen mit F-18-Fluordesoxyglukose (FDG))

- ggf. Angaben zu Name und Dosis einer Zusatzmedikation (z. B. Furosemid, $\beta$-Blocker), ebenso des Applikationsweges

- ggf. Körpergewicht

CT:

- Untersuchte Körperregion(en)

- Angabe, ob eine diagnostische CT erfolgte oder die CT nur zur Schwächungskorrektur und anatomischen Koregistrierung durchgeführt wurde

- Angaben zu Kontrastmittelgabe (Art, Applikationsweg (intravenös, oral), Menge)

- ggf. Angaben zu Kontrastmittelphasen

- ggf. Angaben zur Prämedikation (z. B. bei Kontrastmittelallergie)

- ggf. durchgeführte, zur Befundung verfügbare Rekonstruktionen

MRT:

- Untersuchte Körperregion(en)

- Sequenzprotokoll

- ggf. Angaben zu Kontrastmittelgabe (Art, Applikationsweg (intravenös, oral), Menge)

- ggf. Angaben zu Kontrastmittelphasen

\section{Befund}

Die zum Vergleich herangezogenen bildgebenden Untersuchungen sollen zu Anfang der Befundung benannt werden (mind. Modalität, Untersuchungsgebiet und Datum, ggf. weitere Informationen). Falls solche nicht vorliegen, sollte auch dies vermerkt werden.

Prinzipiell bieten sich zwei übergeordnete Strukturen für den eigentlichen Befundteil an: Zum einen eine separierte, eher systematische Struktur, die den PET und den CT bzw. MRT-Anteil trennt und nacheinander darstellt (Anhang 1), zum anderen eine inte- grierte Struktur, in der der PET und der CT bzw. MRT-Anteil gemeinsam befundet werden (Anhang 2). Dabei ist beiden Ansätzen gemeinsam, dass sie zum Zweck der Übersichtlichkeit in anatomischer Abfolge bzw. nach anatomischen Regionen strukturiert sein sollten. Eine identische Benennung der Läsionen in der PET und dem CT bzw. MRT-Anteil bei separierter Befundung ermöglicht eine einfache Zuordnung der Läsionen in der jeweiligen Modalität.

Im PET-Anteil des Befundes soll die Lokalisation und Intensität (z. B. anhand des SUV) von Läsionen benannt werden, ggf. auch deren Ausdehnung. Die Intensität der Akkumulation des Radiopharmakons kann hierbei visuell graduiert werden (z. B. als gering, mäßig, intensiv) und/oder gegenüber der Radiopharmakonaufnahme im Hintergrund oder einer Referenz (z. B. Leber) verglichen werden [4]. Allerdings variieren Kriterien für die visuelle Interpretation möglicherweise abhängig vom eingesetzten Radiopharmakon oder der untersuchten Entität, und qualitative Kriterien sollten im jeweiligen Anwendungskontext klar definiert sein. Beispielhaft sollen hier die etablierte Lugano-Klassifikation für die FDG PET beim Hodgkin- und Non-Hodgkin-Lymphom, die das Deauville-5-Punkte-Scoring-System bei Lymphomen inkorporiert [19, 20], und die vorgeschlagenen Prostate Cancer Molecular Imaging Standardized Evaluation (PROMISE) Kriterien für die PET mit Liganden des prostataspezifischen Membranantigens (PSMA) beim Prostatakarzinom genannt werden [21].

Im CT bzw. MRT-Anteil des Befundes sollen alle relevanten Befunde beschrieben werden, auch wenn diese keine Aufnahme des Radiopharmakons zeigen. Die Größe von Tumorläsionen soll angegeben werden. Zudem sollen in allen Modalitäten in gleicher Weise auch relevante Zusatzbefunde benannt werden.

Im Falle von Verlaufsuntersuchungen, insbesondere bei einer Beurteilung des Therapieansprechens, soll der Befundaufbau der Folgeuntersuchung idealerweise den Befundaufbau der Voruntersuchung spiegeln, um so die Vergleichbarkeit zu erleichtern [4]. Insbesondere sollte eine klare Aussage zu neu aufgetretenen Läsionen getroffen werden, welche dann eine Progression begründen können. Ebenso soll die Aufnahme des Radiopharmakons bzw. Größenentwicklung maligner Läsionen unter Therapie verglichen und benannt werden. Hierbei ist insbesondere auch ein gemischtes Ansprechen mit diskordanten Befunden herauszustellen, insbesondere wenn dies unterschiedliche zugrundeliegende Pathologien bedeuten könnte. Beispiele für eine standardisierte Befundung des Therapieansprechens sind für die F-18-FDG PET die PET Response Criteria in Solid Tumors (PERCIST)-Kriterien für solide Tumore [22], die Lugano-Klassifikation unter Anwendung des Deauville-5-Punkte-Scoring-Systems bei Lymphomen [19, 20] und die Response Evaluation Criteria in Solid Tumors (RECIST) für die morphologische Bildgebung [23]. Die Reliabilität semiquantitativer Parameter in der PET wird in der Beurteilung des Therapieansprechens von verschiedenen biologischen und technischen Faktoren beeinflusst [24].

Im Befund sollen außerdem Limitationen der durchgeführten Untersuchung benannt werden, wenn sie die diagnostische Aussagekraft beeinflussen könnten. Hierzu zählen für den Bereich der PET beispielsweise ein erhöhter Nüchternblutzuckerwert, eine anormale Biodistribution, die Aktivierung braunen Fettgewebes bei der F-18-FDG PET, Bewegungsartefakte oder Rekonstruk- 
tionsartefakte, für den Bereich der CT oder MRT beispielsweise Metallartefakte und Bewegungsartefakte.

\section{Zusammenfassende Beurteilung}

Die zusammenfassende Beurteilung dient der integrativen Interpretation der wesentlichen Befunde im klinischen Kontext und soll diese nicht bloß rekapitulieren. Sie ist wesentlicher Ausdruck der ärztlichen Expertise des befundenden Arztes und beeinflusst das Patientenmanagement und das Outcome [12, 16, 25]. In der Beurteilung soll ein klarer Bezug zur klinischen Fragestellung hergestellt werden und diese möglichst direkt beantwortet werden. Hierbei ist eine definitive Diagnose anzustreben, die klar ein normales gegenüber einem pathologischen Ergebnis abgrenzt. Falls sinnvoll, soll eine in ihrer Wahrscheinlichkeit priorisierte Angabe von möglichen Differentialdiagnosen erfolgen. Falls erforderlich, können weitere, z. B. auch bildgebende, diagnostische Maßnahmen empfohlen werden, um Befunde zu verifizieren oder weiter einzugrenzen. Falls möglich (z. B. beim nicht-kleinzelligen Lungenkarzinom), soll abschließend ein klinisches Tumorstadium (cTNM) angegeben werden, um die Ergebnisse der Hybriduntersuchung zusammenzufassen und zu systematisieren. Bei einer Beurteilung des Therapieansprechens soll -falls im klinischen Kontext sinnvoll oder erforderlich- eine konklusive Beurteilung erfolgen, die sich an kontextabhängigen, standardisierten Kriterien orientiert (z. B. im Fall der Beurteilung des Therapieansprechens bei Lymphomen an den Lugano-Kriterien [19] und/oder eine onkologisch übliche Terminologie gebraucht (z. B. Progression, stabile Erkrankung, partielle oder komplette Remission)) und die Kommunikation mit dem zuweisenden Arzt erleichtert. Bei der Verwendung einer durch Kriterien geprägten Terminologie (z. B. stabile Erkrankung) ist auf eine kriterienadäquate Befundung und Interpretation zu achten (z. B. Vergleich gegenüber der Ausgangsuntersuchung und nicht gegenüber der letzten Verlaufsuntersuchung).

Falls dringliche, z. B. vital relevante Befunde vorliegen, sollen diese unverzüglich an den zuweisenden Arzt kommuniziert werden. Hierbei empfiehlt sich eine Dokumentation, gegenüber wem und zu welchem Zeitpunkt diese Kommunikation erfolgte.

\section{Schlussfolgerungen}

Seit Einführung der PET/CT im Jahr 2001 bzw. der PET/MRT im Jahr 2010 ist die PET als Hybridbildgebung und Instrument einer bildgesteuerten Präzisionsmedizin zunehmend zu einem Versorgungsstandard in der Onkologie geworden [1]. Die zentrale Schnittstelle in der Kommunikation zwischen den zuweisenden onkologisch tätigen Ärzten und dem befundenden Nuklearmediziner und/oder Radiologen stellt der schriftliche Befund dar, der das Patientenmanagement und Outcome beeinflusst, aber auch juristisch-relevante Dokumentation der erbrachten Leistung und ärztlichen Beurteilung der erhobenen Befunde ist [9, 11, 12]. Ein qualitativ hochwertiger, strukturierter schriftlicher Befund verdeutlicht auch den Mehrwert einer Hybridbildgebung. Die hier dargelegten Empfehlungen zur strukturierten Befunderstellung sollen eine weitere Standardisierung und Harmonisierung onkologischer Befunde im Kontext einer Hybridbildgebung fördern und so die Kommunikation mit den zuweisenden Ärzten vereinfachen und die Akzeptanz und Wertschätzung der onkologischen Hybridbildgebung weiter unterstützen.

\section{Funding}

Mit finanzieller Unterstützung der Arbeitsgemeinschaft Bildgebung in der Onkologie (ABO) der Deutschen Krebsgesellschaft (DKG) (Durchführung des Workshops).

\section{Interessenkonflikt}

Die Autorinnen/Autoren geben an, dass kein Interessenkonflikt besteht.

\section{Literatur}

[1] Derlin T, Grünwald V, Steinbach J et al. Molecular Imaging in Oncology Using Positron Emission Tomography. Dtsch Arztebl Int 2018; 115: 175181

[2] Bischof Delaloye A, Carrió I, Cuocolo A et al. White paper of the European Association of Nuclear Medicine (EANM) and the European Society of Radiology (ESR) on multimodality imaging. Eur J Nucl Med Mol Imaging 2007; 34: 1147-1151

[3] Gourtsoyiannis N, McCall I, Reiser M et al. White paper of the European Society of Radiology (ESR) and the European Association of Nuclear Medicine (EANM) on multimodality imaging. Eur Radiol 2007; 17: 19261930

[4] Boellaard R, Delgado-Bolton R, Oyen W] et al. FDG PET/CT: EANM procedure guidelines for tumour imaging: version 2.0. Eur J Nucl Med Mol Imaging 2015; 42: 328-354

[5] Umutlu L, Beyer T, Grueneisen JS et al. Whole-Body [18F]-FDG-PET/MRI for Oncology: A Consensus Recommendation. Nuklearmedizin 2019; 58 : 68-76

[6] Umutlu L, Beyer T, Grueneisen JS et al. Whole-Body [18F]-FDG-PET/MRI for Oncology: A Consensus Recommendation. Rofo 2019; 191: 289-297

[7] Beyer T, Hicks R, Brun C et al. An international survey on hybrid imaging: do technology advances preempt our training and education efforts? Cancer Imaging 2018; 18: 15

[8] Beyer T, Hicks RJ, Freudenberg LS. Life is not black and white, nor just Shades of Gray. Eur J Nucl Med Mol Imaging 2018; 45: 816-821

[9] Freudenberg LS, Hicks R], Beyer T. An International Survey on Clinical Reporting of PET/CT Examinations: A Starting Point for Cross-Specialty Engagement. J Nucl Med 2019; 60: 480-485

[10] Gatidis S, Beyer T, Becker M et al. State of affairs of hybrid imaging in Europe: two multi-national surveys from 2017. Insights Imaging 2019; 10: 57

[11] Niederkohr RD, Greenspan BS, Prior JO et al. Reporting guidance for oncologic 18F-FDG PET/CT imaging. J Nucl Med 2013; 54: 756-761

[12] Coleman RE, Hillner BE, Shields AF et al. PET and PET/CT reports: observations from the National Oncologic PET Registry. J Nucl Med 2010; 51 : 158-163

[13] Beyer T, Czernin J, Freudenberg LS. Variations in clinical PET/CT operations: results of an international survey of active PET/CT users. J Nucl Med 2011; 52: 303-310

[14] Mayerhoefer ME, Prosch $H$, Beer L et al. PET/MRI versus PET/CT in oncology: a prospective single-center study of 330 examinations focusing on implications for patient management and cost considerations. Eur J Nucl Med Mol Imaging 2020; 47: 51-60

[15] Pfannenberg C, Gueckel B, Wang L et al. Practice-based evidence for the clinical benefit of PET/CT-results of the first oncologic PET/CT registry in Germany. Eur J Nucl Med Mol Imaging 2019; 46: 54-64 
[16] Hillner BE, Siegel BA, Liu D et al. Impact of positron emission tomography/computed tomography and positron emission tomography (PET) alone on expected management of patients with cancer: initial results from the National Oncologic PET Registry. J Clin Oncol 2008; 26: 21552161

[17] Stegger L, Schäfers M, Weckesser $M$ et al. EANM-ESR white paper on multimodality imaging. Eur ] Nucl Med Mol Imaging 2008; 35: 677-680

[18] Vereinbarung von Qualitätssicherungsmaßnahmen nach § 135 Abs. 2 SGB V zur diagnostischen Positronenemissionstomographie, diagnostischen Positronenemissionstomographie mit Computertomographie (QS-Vereinbarung PET, PET/CT) vom 01.07.2016 in der ab dem 01.04.2019 geltenden Fassung. www.kbv.de/media/sp/PET_CT.pdf (abgerufen am 27.11.2019)

[19] Cheson BD, Fisher RI, Barrington SF et al. Recommendations for initial evaluation, staging, and response assessment of Hodgkin and nonHodgkin lymphoma: the Lugano classification. J Clin Oncol 2014; 32: 3059-3068

[20] Barrington SF, Qian W, Somer EJ et al. Concordance between four European centres of PET reporting criteria designed for use in multicen- tre trials in Hodgkin lymphoma. Eur ] Nucl Med Mol Imaging 2010; 37: 1824-1833

[21] Eiber M, Herrmann K, Calais J et al. Prostate Cancer Molecular Imaging Standardized Evaluation (PROMISE): Proposed miTNM Classification for the Interpretation of PSMA-Ligand PET/CT. J Nucl Med 2018; 59: 469478

[22] Wahl RL, Jacene $\mathrm{H}$, Kasamon $\mathrm{Y}$ et al. From RECIST to PERCIST: evolving considerations for PET response criteria in solid tumors. J Nucl Med 2009; 50 (Suppl. 1): 122S-150S

[23] Eisenhauer EA, Therasse P, Bogaerts J et al. New response evaluation criteria in solid tumours: revised RECIST guideline (version 1.1). Eur J Cancer 2009; 45: 228-247

[24] de Langen AJ, Vincent A, Velasquez LM et al. Repeatability of 18F-FDG uptake measurements in tumors: a metaanalysis. J Nucl Med 2012; 53 : 701

[25] Hillner BE, Siegel BA, Shields AF et al. Relationship between cancer type and impact of PET and PET/CT on intended management: findings of the national oncologic PET registry. J Nucl Med 2008; 49: 1928-1935 